\section{Economic evaluation of whole-genome sequencing in healthy individuals: what can we learn from CEAs of whole-body CT screening?}

To the Editor: The recent study by Bennette et al. ${ }^{1}$ focused on the cost-effectiveness of returning incidental findings after next-generation sequencing but indirectly assessed its costeffectiveness in an exploratory analysis of a scenario of primary screening with genomic sequencing. The limited evidence on the costs/benefits of whole-genome sequencing (WGS) in the general population remains to be resolved before WGS becomes routine. Many previous studies (e.g., by Buchanan et $\mathrm{al}^{2}$ and Phillips et al. ${ }^{3}$ ) have discussed the challenges of conducting economic evaluations of genomic technologies.

Whole-body computed tomography (WBCT) for screening healthy individuals provides an intriguing comparison to WGS in the general population. WBCT uses X-rays to produce cross-sectional images of the human body in order to detect a tumor or other abnormality. Clinical scenarios in which WBCT is invaluable include disease diagnosis, evaluation after trauma, and monitoring response to oncologic therapy. WBCT is also marketed as a preventive diagnostic for healthy individuals. Under such use, there are key similarities between WBCT screening and WGS: (i) WBCT may incidentally detect benign lesions that may lead to costly additional evaluation with potential physical and mental harm; (ii) because the majority of individuals who undergo screening do not have the disease in question, the costs and potential risks are spread across a wider population; and (iii) costs and potential harms generally occur in the short term, but any benefits are typically realized in the long term.

I reviewed the literature for economic evaluation of WBCT screening; few were identified. Beinfeld et al. ${ }^{4}$ directly examined the cost-effectiveness of WBCT screening in asymptomatic individuals for signals for risks of several diseases (ovarian cancer, pancreatic cancer, lung cancer, liver cancer, kidney cancer, colon cancer, abdominal aortic aneurysm, and coronary artery disease); their methods may provide insights for addressing the key challenges of conducting economic evaluations of WGS in healthy individuals.

Approaches taken by Beinfeld et al. ${ }^{4}$ highlighted several major gaps in knowledge about WGS that are research priorities. Appropriately, Beinfeld et al. included a broad range of costs and resource use. They were able to identify and use the average advertised price of WBCT. Estimates of health-care costs related to disease management were identified from the literature. For instance, costs of routine care for coronary artery disease including predicted cost savings due to prevention of myocardial infarction by statin therapy. Costs of follow-up testing for patients with false-positive scans and costs of treating complications were derived from Medicare reimbursement rates (e.g., cost of angiography). Disease-specific sensitivity and specificity of WBCT for the eight included diseases were identified from the literature and cancer stage-specific 5-year survival estimates from the Surveillance, Epidemiology and End Results program. Many similar model inputs for WGS (including performance of WGS for many diseases) are not well known and still need to be collected.

Several approaches used by Beinfeld et al. ${ }^{4}$ may or may not be appropriate for economic evaluations of WGS, but at a minimum these are valuable for discussion by scientists in the precision medicine field. First, the authors used "no screening" as the comparator for WBCT screening. For WGS, possible comparators include no sequencing, multiple single-gene tests, and gene panels. It is important to consider the current standard practice as well as emerging practices. Second, they did not take into account cost variations for WBCT between providers/facilities. Cost variations for WGS between laboratories, regions, or countries are not well studied, but cost used in the analysis would influence cost-effectiveness estimates. Third, they allowed only false-positive results and workup costs for the eight conditions included in the study. Compared with WBCT screening, WGS is capable of detecting risk of a greater number of diseases, including rare genetic diseases, but as a result may produce a higher number of false positives that necessitate additional tests and costs; these could negatively affect cost-effectiveness.

Importantly, Beinfeld et al. did not consider secondary findings of WBCT screening because of the low prevalence of other conditions and because false-positive and true-positive results may have offsetting effects. For WGS, however, much attention has been paid to incidental findings because they may provide clinical benefits, which is recognized as a methodological challenge for the economic evaluation of WGS. Compared with WBCT screening, an added complexity is our currently limited (but accumulating) knowledge of genes in the human genome. Only a quarter of the $\sim 23,000$ genes in the human genome are described in comprehensive databases such as the Human Gene Mutation Database (http://www.hgmd.org). Therefore, we are guaranteed to find thousands of variants of unknown significance that are not related to a patient's current health when we test the whole genome.

Fourth, Beinfeld et al. assumed that diseases would be detected at earlier stages by WBCT screening than if they had been detected incidentally and/or on the basis of the development of symptoms, and they made assumptions about 


\section{LETTER TO THE EDITOR}

patient and clinician behaviors (e.g., patients with a positive result would undergo additional testing; the clinical course for patients with a false-negative result would be similar to those had no WBCT). Whether using similar assumptions in economic evaluations of WGS is appropriate deserves consideration, particularly given the paucity of data on patient and clinician behavior after knowing genomic sequencing results.

Fifth, Beinfeld et al. did not consider health-related quality of life because of insufficient data; they argued that not only disease-specific quality-of-life estimates for all eight diseases would be needed but also quality-of-life estimates for the different stages of the six cancers would need to be included. Similarly, data on health-related quality of life for WGS are scarce. Sixth, cancer risk due to radiation exposure was not adjusted because WBCT screening was assumed to be a onetime use, which might be appropriate. WGS use is not associated with such harms; however, it may have negative impacts on personal utility, such as increased anxiety because of falsepositive results or incidental findings. Interestingly, because anxiety as a result of false-positive tests may offset the benefit to quality of life from knowing one is disease free, Beinfeld et al. did not account for personal utility. Finally, Beinfeld et al. assumed independence of diseases, which overlooks the fact that many conditions may be related. A key methodological challenge is how to assess the joint impact of multiple conditions on the cost-effectiveness of WGS.

In summary, several approaches used in economic evaluations of WBCT screening may deserve discussion as we refine methodological approaches to conducting economic evaluations of WGS.

\section{ACKNOWLEDGMENTS}

The author thanks Michael P. Douglas and Kathryn A. Phillips (University of California at San Francisco Center for Translational and Policy Research on Personalized Medicine) for their help and feedback on this article.

\section{DISCLOSURE}

The author declares no conflict of interest.

\section{Christine Y. Lu, MSc, $P h D^{1}$}

${ }^{1}$ Department of Population Medicine, Harvard Medical School and Harvard Pilgrim Health Care Institute, Boston, Massachusetts, USA. Correspondence: Christine Y. Lu (christine_lu@harvardpilgrim.org)

\section{REFERENCES}

1. Bennette CS, Gallego CJ, Burke W, Jarvik GP, Veenstra DL. The cost-effectiveness of returning incidental findings from next-generation genomic sequencing. Genet Med 2015;17:587-595.

2. Buchanan J, Wordsworth S, Schuh A. Issues surrounding the health economic evaluation of genomic technologies. Pharmacogenomics 2013;14:1833-1847.

3. Phillips KA, Ann Sakowski J, Trosman J, Douglas MP, Liang SY, Neumann P. The economic value of personalized medicine tests: what we know and what we need to know. Genet Med 2014;16:251-257.

4. Beinfeld MT, Wittenberg E, Gazelle GS. Cost-effectiveness of whole-body CT screening. Radiology 2005;234:415-422.

Advance online publication 3 December 2015. doi:10.1038/gim.2015.159 